\title{
A case of boundedness in Littlewood's problem on oscillatory differential equations
}

\section{G.R. Morris}

It is shown that all solutions of $\ddot{x}+2 x^{3}=p(t)$ are bounded, the notation indicating that $p$ is periodic. It is not necessary to have a small parameter multiplying $p$. The essential step is to show by appeal to Moser's theorem that, under the mapping (of the initial-value plane) which corresponds to the equation, there are invariant simple closed curves. This implies also that there is an uncountable infinity of almostperiodic solutions and, for each positive integer $m$, an infinity of periodic solutions of least period $2 m \pi$ ( $2 \pi$ being taken as the least period of $p$ ).

It is suggested that for a large class of equations the same attack would show all solutions of $\ddot{x}+g(x)=p(t)$ bounded. However, in order to show the method clearly, no generalisation is attempted here.

\section{Introduction}

Littlewood proposes, as Problem 29 in [6], the consideration of the equation

$$
\ddot{x}+g(x)=p(t),
$$

Received 30 October 1975. The author would like to thank Dame Mary Cartwright, Professor J.E. Littlewood, and Professor Jürgen Moser for comments on a draft of this paper. A question from Professor V.A. Pliss led him to rethink the treatment of Theorems 2 and 3. 
where $g(x) \operatorname{sgn} x \rightarrow \infty$ as $x \rightarrow \pm \infty$ and

(a) $p(t)$ is bounded, or

(b) $p(t)$ is periodic.

As he remarks, it has been conjectured that if either

$$
g(x) / x \rightarrow \infty
$$

or $g(x) / x \rightarrow 0$ then there are no unbounded solutions. In the form $(1.2)(a)$, that is, when all $g(x)$ satisfying (1.2) and all bounded $p(t)$ are admitted, the conjecture is certainly false: Lit+lewood himself has shown in [4] that, given a fairly general $g(x)$, there exist associated bounded $p(t)$ for which (1.1) has an unbounded solution. Further, what is more important for us, he has shown in [5] how to construct functions $g(x)$ with each of which can be associated a periodic $p(t)$ such that (1.1) has an unbounded solution. In his construction any $g(x)$ is approximately equal to $4 x^{3}$ but is discontinuous. There is a discussion of the relation of Littlewood's construction to the results of this paper in section 7.

Evidently the equation

$$
\ddot{x}+2 x^{3}=p(t)
$$

is a very special case of (1.1) with $g$ satisfying (1.2). When (in Section 2) we give a general description of the method employed here, it will be possible to show why the choice $g(x)=2 x^{3}$ is forced upon us if we wish to benefit from all the simplifications in the calculations that can be foreseen. Nevertheless, I suggest that under suitable conditions on $g$ (concerning smoothness and regularity of increase) estimations like those of Section 5 could be carried through; in particular, I should expect that, whenever $g(x)$ is a polynomial of odd degree greater than 1 , all solutions of (1.1) will be bounded. It might be remarked here that, to avoid special constructions of auxiliary functions, we use results from the theory of elliptic functions. However, if Lemma 1 is accepted, the paper is independent of previous knowledge of that theory and it will be observed that the special notations of elliptic functions do not occur.

THEOREM 1. If $p(t)$ has period $2 \pi$ and is piecewise continuous, then every solution of (1.3) is bounded. Further, there is a $B$, depending only on $\max |p(t)|$, such that every solution satisfies 


$$
\sup \left\{\dot{x}^{2}(t)+x^{4}(t)\right\}^{\frac{1}{4}}-\inf \left\{\dot{x}^{2}(t)+x^{4}(t)\right\}^{\frac{3}{4}} \leq B
$$

The work which leads to Theorem 1 allows us to establish the existence of interesting special solutions. We write $\omega$ for the constant defined by (3.3) and (3.4) below, and for a typical solution write $\xi=x(0)$ and $\eta=\dot{x}(0)$. It will be observed that the $\phi$ in Theorem 3 is Euler's totient function and not the function $\phi$ of Sections 4 and 5 . We shall prove

THEOREM 2. There is in $[0,1]$ a set $\Lambda$ of irrationals such that

(i) $\mathrm{m} \Lambda=1$,

(ii) if $\lambda \in \Lambda$, there is a $\mu^{*}=\mu^{*}(\lambda)$ with the property that, for every integer $\mu$ with $\mu \geq \mu^{*}$, there is an almostperiodic solution of (1.3) having basic frequencies $2 \pi$ and $2 \pi \lambda$ and satisfying

$$
(\mu+1)^{4} \omega^{4}<\eta^{2}+\xi^{4}<(\mu+2)^{4} \omega^{4} .
$$

THEOREM 3. If $p(t)$ has least period $2 \pi$, there is a $k^{*}$ such that for every integer $\kappa$ with $\kappa \geq \kappa^{*}$ there are, for every positive integer $m$, at least $2 m \phi(m)$ solutions of (1.3) having least period $2 m \pi$ and satisfying

$$
\kappa^{4} \omega^{4}<\eta^{2}+\xi^{4}<(\kappa+2)^{4} \omega^{4}
$$

There is one comment on these results. Since they all follow from results which hold provided some quantity is "sufficiently small" and there is at present no prospect of estimating the smallness required, we must think of $B, \mu^{*}$, and $K^{*}$ as not constructible. This is to be compared with results which are obtained by calculations with error terms. In such work, although it would be tedious, we should expect to be able to estimate quantities corresponding to $k^{*}$ above. (For example, in Theorem 6 of my [8], which resembles Theorem 3 of this paper, we should expect to be able to estimate what is there called $p$.)

\section{General description of the method}

We shall show that in the phase (or initial-value) plane there are arbitrarily large closed curves invariant under $T$, the Poincaré map corresponding to (1.3). The essential point is that we express the phase 
plane as the union of a disc and a sequence of (non-circular) annuli $\left\{A_{\mu}\right\}$, and then transform each large $A_{\mu}$ into a standard circular annulus $A^{*}$ - For each large $\mu$, we are thus led to define on $A^{*}$ a map $T_{\mu}^{*}$ which (in the new coordinates) corresponds to the restriction of the map $T$ to $A_{\mu}$. Although (1.3) does not contain a small parameter, $T_{\mu}^{*}$ does, namely $1 / \mu$ for large $\mu$. As $\mu \rightarrow \infty, T_{\mu}^{*}$ approaches a twist mapping and, if we obtain suitable estimates on derivatives, Moser's theorem gives us that, for large $\mu$, there is a closed curve in $A^{*}$ which is invariant under $T_{\mu}^{*}$, a result which, when reinterpreted, shows us there is, for each large $\mu$, a simple closed curve in $A_{\mu}$ which is invariant under $T$.

From the existence of these curves we obtain Theorem 1 . With some extra detail about such curves we obtain Theorem 2 and, by appeal to Birkhoff's (Poincaré's Last Geometric) Theorem, Theorem 3.

In the above there was no mention of the special choice $g(x)=2 x^{3}$. If we think of functions $g$ satisfying (1.2) it is essential that we can make the assertions:

(Al) large solutions of (I.I) are well approximated by solutions of

$$
\ddot{z}+g(z)=0
$$

(A2) all large solutions of (2.1) are periodic, and if $P(h)$ denotes the least period of a solution with extreme values $h>0$ and $-h^{\prime}<0$ then

$$
P(h) \rightarrow 0
$$

as $h \rightarrow \infty$.

of these (A2) follows readily from the first integral

$$
\dot{z}^{2}+2 G(z)=\text { const },
$$

which leads to the formula

$$
P(h)=\sqrt{ } 2 \int_{-h}^{h}, \frac{d z}{V\{G(h)-G(z)\}},
$$

and (AI) by using (2.3) as a basis for successive approximations. (As 
usual, $G$ is defined by

$$
G(x)=\int_{0}^{x} g(u) d u
$$

Since, in using (A1) and (A2), we need quite detailed information on the quality of the approximation of the solutions of (1.1) by those of (2.I) it is natural to look for restrictions which we can lay on $g$. The following are useful:

(RI) $g$ is odd;

(R2) $g(x) \operatorname{sgn} x>0$ for $x \neq 0$;

(R3) $g(x)=k|x|^{\alpha} \operatorname{sgn} x$, where $\alpha>1, k>0$;

(R4) $g$ is analytic in a strip containing the real axis;

(R5) $g$ is a polynomial, with deg $g \leq 3$.

From (RI) we can deduce that $h^{\prime}=h$, from (R2) that all non-trivial solutions of (2.1) are periodic and from (R3) that (2.4) is simplified to

$$
P(h)=C h^{(1-\alpha) / 2} \text {, }
$$

which gives the strengthening and simplification of (2.2) that $P(h)$ tends monotonically to 0 . The useful consequences of (R4) are that $z(t)$ becomes an analytic function of $t$, whence complex-variable methods are available for the estimations, and of (R5) that the formulae of elliptic functions can be quoted. It is clear that all these consequences can hold simultaneously, and that for this we must have $g(x)=k x^{3}$. If, further, we recognise that the most useful standardisation of (2.3) is

$$
\dot{z}^{2}+z^{4}=h^{4}
$$

we are led to the choice $k=2$, that is, to $g(x)=2 x^{3}$.

\section{Properties of the comparison equation}

When we make the choice $g(x)=2 x^{3}$, the comparison equation (2.1) becomes

$$
\ddot{z}+2 z^{3}=0
$$


We have already indicated the simpler (real-variable) properties of (3.1) when we discussed (2.1) and need only introduce enough notation to s.tate them clearly. The deeper (complex-variable) properties we shall quote.

We write $z(t ; \xi, \eta)$ for the solution of (3.1) with $z(0)=\xi$ and $\dot{z}(0)=n$, and (2.3) then becomes

$$
\dot{z}^{2}(t ; \xi, \eta)+z^{4}(t ; \xi, \eta)=h^{4},
$$

where $h$ is defined by

$$
h^{4}=n^{2}+\xi^{4}
$$

subject to $h \geq 0$. From (3.2) it follows that any solution with $h>0$ is periodic, its least period being $2 \pi / h$, where

$$
\frac{1}{2} \sigma=\int_{0}^{1} \frac{d u}{v\left(1-u^{4}\right)} .
$$

Since (3.1) is autonomous and involves $z$ only as a power, any solution can be simply expressed in terms of some standard solution. We choose

$$
c(t)=z(t ; \omega, 0) \text {, }
$$

where $\omega$ is defined by

$$
\omega=\omega / \pi
$$

we write $s(t)=\dot{c}(t)$.

LEMMA 1. (i) The functions $c(t)$ and $s(t)$ have $2 \pi$ as least real period. They satisfy

$$
\begin{aligned}
\dot{s}(t) & =-2 c^{3}(t), \\
s\left(\frac{1}{2} \pi\right) & =-\omega^{2}, \\
s^{2}(t)+c^{4}(t) & =\omega^{4} .
\end{aligned}
$$

(ii) For every $\rho>0$ and every real $\phi, \rho c(\rho t+\phi)$ is a solution of (3.1) for all real $t$. Conversely, if $z(t)$, not identically 0 , is a real solution of (3.1) it can be expressed in this form, $\rho$ being determined uniquely and $\phi$ modulo $2 \pi$. If $\rho$ is an integer this solution has $2 \pi$ as a period.

(iii) The functions $c$ and $s$ are analytic on the real axis. More 
generally, if $u$ is a complex variable $c(u)$ and $s(u)$ are analytic in $|\operatorname{Im} u|<\frac{3}{2} \pi$.

For (iii), we quote results from Whittaker and Watson [10]. A rearrangement of their work on p. 524 shows that

$$
c(u)=\operatorname{cn}(\omega \sqrt{ } 2 u, 1 / \sqrt{ } 2),
$$

a function which is analytic in

$$
|\operatorname{Im}(\omega \sqrt{2} u)|<K_{0}=\pi / \sqrt{2},
$$

that is, in

$$
|\operatorname{Im} u|<\frac{1}{2} \pi
$$

\section{Geometrical representations of solutions}

We write $(1.3)$ as the system

$$
\dot{x}=y, \dot{y}=-2 x^{3}+p(t),
$$

and then, as foreshadowed, define the mapping $T$ of the phase plane into itself by

$$
T(\xi, \eta)=(x(2 \pi ; \xi, \eta), y(2 \pi ; \xi, \eta)) .
$$

We have of course denoted by $(x(t ; \xi, n), y(t ; \xi, n))$ the solution of (4.I) with $x(0)=\xi$ and $y(0)=n$; it will sometimes be convenient to write $T(\xi, \eta)$ as $\left(\xi_{1}, \eta_{1}\right)$. As is familiar, to a fixed point of $T$ corresponds a periodic solution of (4.1). As mentioned in Section 2 , our concern will be the search for simple closed curves invariant under $T$.

However useful it is to talk in terms of the plane mapping $T$, it is still essential for us to recall that an unambiguous geometrical description of a solution of (4.1) is as a curve in $(t, x, y)$-space. We shall describe $\Sigma$, parametrised as

$$
(t, x(t ; \xi, \eta), y(t ; \xi, n))
$$

as the trajectory corresponding to $(x(t ; \xi, n), y(t ; \xi, \eta))$. We shall also say that $\Sigma$ is the trajectory arising from the point $(0, \xi, n)$.

So far, we have said nothing about the interval of $t$ for which $x(t ; \xi, \eta)$ is defined. In fact, for any $(a, b), x(t ; a, b)$ is defined for $-2 \pi \leq t \leq 2 \pi$ and hence, by repeated use of this result, $x(t ; \xi, \eta)$ 
is defined for all $t$. Rather than pause to prove this, we assert that it follows from the general observation that $x(t ; \xi, \eta)$ is usefully approximated in, say, $[-2 \pi, 2 \pi]$ by $z(t ; \xi, n)$ when $h$ is large, and that this itself can be established by using the approximate first integral

$$
y^{2}(t ; \xi, n)+x^{4}(t ; \xi, n)=h^{4}+2 \int_{0}^{t} y(\tau) p(\tau) d \tau \text {. }
$$

We shall in passing assume other simple consequences of the above observation. (With a different emphasis and with much more detail than is needed here, the application of the approximate first integral is explored in [7].)

The existence of $x(t ; \xi, \eta)$ for all $t$ is of course reflected in the existence of $\Sigma$ for all $t$. Corresponding to the unique determination of a solution of (4.1) by its initial values we have the geometrical property that if $\Sigma$ and $\Sigma^{\prime}$ arise from distinct points $(0, \xi, \eta)$ and $\left(0, \xi^{\prime}, n^{\prime}\right)$ they have no common point. As an obvious development of this property we can say that if, in the plane $t=0, \Gamma$ is an arc and $P$ a point off $\Gamma$, then the trajectories arising from points of $\Gamma$ form a piece of surface which is not met by the solution curve arising from $P$. The following specialisation of this remark will be an essential tool.

LEMMA 2. (i) If $\Gamma$ is a simple closed curve in the plane $t=0$ then the trajectories arising from its points form a tube $T$ which meets any plane $t=\tau$ in a simple closed curve.

(ii) If $P$ is a point of the plane $t=0$, not on $\Gamma$, then the trajectory $\Sigma$ arising from $P$ will not meet $T$. In particular, if $P$ is inside $\Gamma$ then $\Sigma$ is wholly inside $T$.

Suppose now that we have found some simple closed curves which are invariant under $T$. From any such curve $\Gamma$ in the phase plane (which we identify with the plane $t=0$ of $(t, x, y)$-space) there arises a tube, $T$ say. Since $p$ is periodic, $T$ is easy to describe: the piece between the planes $t=2 n \pi$ and $t=2(n+1) \pi$ is congruent to that between the planes $t=0$ and $t=2 \pi$. To prove this we need only remark

(i) that the invariance of $\Gamma$ under $T$ is expressed in the three-dimensional representation by the fact that $T$ cuts 
$t=2 \pi$ in a curve directly above $\Gamma$ (if we think of the $t$-axis as vertical), and

(ii) that, because of the periodicity of (1.3) with respect to $t$, this piece of $T$ is indeed repeated.

It will be observed that, on a tube $T$ arising from an invariant closed curve, $y^{2}+x^{4}$ is bounded for all $t$ and that $T$ separates $(t, x, y)-$ space.

Our principal tool in proving Theorem 1 will be the following result.

LEMMA 3. There is an integer $\mu_{1}$ such that, for every integer $\mu$ with $\mu \geq \mu_{1}$, there is, in the annulus

$$
A_{\mu}:(\mu+1)^{4} \omega^{4} \leq n^{2}+\xi^{4} \leq(\mu+2)^{4} \omega^{4} \text {, }
$$

a simple closed curve which encloses $(0,0)$ and is invariant under $T$.

In the remainder of this section we shall show that Lemma 3 is equivalent to Lemma 4 (which is enunciated below and established in Section 6).

We shall, as indicated in Section 2, transform each $A_{\mu}$ (at least for large $\mu$ ) into a standard circular annulus $A^{*}$. Corresponding to any solution $(x(t), y(t))$ of (4.1) we can imitate (ii) of Lemma 1 and attempt to define $\rho(t)$ and $\phi(t)$ by

$$
\left\{\begin{array}{l}
x(t)=\rho(t) \cdot c[\rho(t) t+\phi(t)], \\
y(t)=\rho^{2}(t) \cdot s[\rho(t) t+\phi(t)] .
\end{array}\right.
$$

Provided that, for all $t$ in $[0,2 \pi], y^{2}(t)+x^{4}(t) \neq 0$, which is certainly the case for large solutions, (4.2) will define a unique positive $\rho(t)$ for $0 \leq t \leq 2 \pi$. Evidently $\phi(0)$ is determined only modulo $2 \pi$, but if a choice of $\phi(0)$ is made and $\phi(t)$ is required to be continuous, then (4.2) will define $\phi(t)$ uniquely for $0 \leq t \leq 2 \pi$. Further, these two functions will enjoy all properties of smoothness that $x(t)$ and $y(t)$ do.

If $x(t)$ here is an abbreviation for the heavier notation $x(t ; \xi, \eta)$ it is evident that 


$$
\xi=\rho(0) \cdot c[\phi(0)] \text { and } n=\rho^{2}(0) \cdot s[\phi(0)] .
$$

If we replace $\rho(t)$ and $\phi(t)$ by the heavier expressions $\rho(t ; \xi, \eta)$ and $\phi(t ; \xi, n)$, we can think of $\xi$ and $n$ as varying (with $n^{2}+\xi^{4}$ large) and if $\phi\left(0 ; \xi_{0}, n_{0}\right)$ is chosen for some convenient $\left(\xi_{0}, n_{0}\right)$, the functions $\rho$ and $\phi$ will depend continuously on $t, \xi$, and $\eta$ (with the proviso that if $(\xi, \eta)$ varies along a path that makes a complete circuit of $(0,0)$ then $\phi(0 ; \xi, \eta)$ and, more generally, $\phi(t ; \xi, \eta)$ will change by $2 \pi)$. Hence we have the possibility of specifying the point $(\xi, n)$ in $A_{\mu}$ by specifying $\rho(0 ; \xi, \eta)$ and $\phi(0 ; \xi, \eta)$, that is, of using these numbers as "pseudo-polar" coordinates. Instead of this, noticing that on the inner boundary of $A_{\mu}$ we have $\rho(0)=\mu+1$ at every point and on the outer $\rho(0)=\mu+2$, we shall use $\rho(0)-\mu$ and $\phi(0)$ as ordinary polar coordinates in an auxiliary plane in which we introduce a briefer notation.

If $(\xi, \eta) \in A_{\mu}$, introduce $\rho(t ; \xi, \eta)$ and $\phi(t ; \xi, \eta)$ as above and define $r,=r(\xi, \eta)$, and $\theta,=\theta(\xi, \eta)$, by

$$
\left\{\begin{array}{l}
r=\rho(0 ; \xi, \eta)-\mu, \\
\theta=\phi(0 ; \xi, \eta) .
\end{array}\right.
$$

It is clear that we obtain a homeomorphism of $A_{\mu}$ onto the standard annulus

$$
A^{*}: 1 \leq r \leq 2 \text {. }
$$

We now define $r_{1},=r_{1}(\xi, n)$, and $\theta_{1},=\theta_{1}(\xi, n)$, by

$$
\left\{\begin{array}{l}
r_{1}=\rho(2 \pi ; \xi, n)-\mu, \\
\theta_{1}=2 \pi\{\rho(2 \pi ; \xi, n)-\mu\}+\phi(2 \pi ; \xi, n),
\end{array}\right.
$$

and then define $T_{\mu}^{*}$ for $(r, \theta) \in A^{*}$ by

$$
T_{\mu}^{*}(r, \theta)=\left(r_{1}, \theta_{1}\right)
$$

We observe that if we needed only to define $T_{\mu}^{*}$ we could use 


$$
\xi=(\mu+r) c(\theta), \quad \eta=(\mu+r)^{2} s(\theta)
$$

and

$$
\xi_{1}=\left(\mu+r_{1}\right) c\left(\theta_{1}\right), \eta_{1}=\left(\mu+r_{1}\right)^{2} s\left(\theta_{1}\right),
$$

and avoid the introduction of the functions $\rho$ and $\phi$. However, for the estimations connected with $T_{\mu}^{*}$ we need to follow the continuous change of $(r, \theta)$ into $\left(r_{1}, \theta_{1}\right)$, and to do this we need to consider $\rho(t)$ and $\phi(t)$.

We can now enunciate Lemma 4 and assert, on the basis of this discussion, that it is indeed equivalent to Lemma 3.

LEMMA 4. There is an integer $\mu_{1}$ such that, for every integer $\mu$ with $\mu \geq \mu_{1}$, there is in the connulus $A^{*}$ a simple closed curve which encloses the pole of coordinates and is invariant under $T_{\mu}^{*}$.

\section{The estimations}

We rewrite $(4.4)$ as

$$
\begin{aligned}
& \theta_{1}=\theta+2 \pi r+F_{\mu}(r, \theta), \\
& r_{1}=r+G_{\mu}(r, \theta) .
\end{aligned}
$$

LEMMA 5. Suppose that $(\xi, n)$ is in $A_{\mu}$ and that $(r, \theta), \rho(t)$, $\phi(t)$, and $\left(r_{1}, \theta_{1}\right)$ are defined as in (4.3), (4.2), and (4.4). Then

$$
\begin{aligned}
& F_{\mu}(r, \theta)=2 \pi(\rho(2 \pi)-\rho(0))+\phi(2 \pi)-\phi(0), \\
& G_{\mu}(r, \theta)=\rho(2 \pi)-\rho(0) .
\end{aligned}
$$

LEMMA 6. The functions $\rho$ and $\phi$ corresponding to a given $\xi$ and $\eta$ in $A_{\mu}$ are determined by

$$
\begin{aligned}
& \dot{\rho}=\frac{1}{2} \omega^{-4} p(t) s(\rho t+\phi) \cdot \rho^{-1}, \\
& \dot{\phi}=-\frac{1}{2} \omega^{-4} p(t)\left\{t s(\rho t+\phi)+c(\rho t+\phi) \cdot \rho^{-1}\right\} \cdot \rho^{-1},
\end{aligned}
$$

subject to $\rho(0)=\mu+r, \phi(0)=\theta$. 
We have from (4.2) that

$$
4 \rho^{3} \dot{\rho}=d\left(y^{2}+x^{4}\right) / d t=2 p(t) y,
$$

which gives us (5.1). Except when $s(\rho t+\phi)=0,(5.2)$ comes from

$$
\rho^{2} s(\rho t+\phi)=y=d[\rho c(\rho t+\phi)] / d t
$$

if we substitute for $\dot{\rho}$ and divide by $\rho s(\rho t+\phi)$. In the excepted case we know $c(\rho t+\phi) \neq 0$ and we can obtain the equation from

$$
-2 \rho^{3} c^{3}(\rho t+\phi)+p(t)=-2 x^{3}+p(t)=d\left[\rho^{2} s(\rho t+\phi)\right] / d t
$$

if we substitute for $\rho$, use (3.5) and (3.6) and divide by $2 \rho^{2} c^{3}(\rho t+\phi)$

LEMMA 7. Suppose that, in the differential equations (5.1) and (5.2), $\rho$ and $\dot{\phi}$ are alzowed to be complex, $t$ always being real. If, for $\rho(0)$ and $\phi(0)$ in a domain $\Delta, \rho(t)$ and $\phi(t)$ are defined for $0 \leq t \leq 2 \pi$, then $\rho(2 \pi)$ and $\phi(2 \pi)$ depend analytically on $\rho(0)$ and $\phi(0)$.

Since $p$ is piecewise continuous on $[0,2 \pi]$, there is a dissection

$$
0=t_{0}<t_{1}<\cdots<t_{n}=2
$$

such that $p$ is continuous on each $\left(t_{v-1}, t_{v}\right)$ and $p(t)$ tends to a limit as $t$ tends to either endpoint.

If $\rho(0)$ and $\phi(0)$ vary in $\Delta$ we can write

$$
\left(\rho\left(t_{1}\right), \phi\left(t_{1}\right)\right)=U_{1}\left(\rho\left(t_{0}\right), \phi\left(t_{0}\right)\right),
$$

where by [3], Theorem 8.4 , page $36, U_{1}$ is analytic. Similarly we can write

$$
\left(\rho\left(t_{2}\right), \phi\left(t_{2}\right)\right)=v_{2}\left(\rho\left(t_{1}\right), \phi\left(t_{1}\right)\right)
$$

with $U_{2}$ analytic, and so on, and obtain finally

$$
(\rho(2 \pi), \phi(2 \pi))=U_{n} U_{n-1} \ldots U_{2} U_{1}(\rho(0), \phi(0)) .
$$

Since the composition of analytic transformations gives an analytic transformation our result is proved. 
LEMMA 8. Suppose that in (5.1) and (5.2), $\rho$ and $\phi$ are allowed to be complex, $t$ always being real. Given $\gamma>0$, there is an integer $K(\gamma)$ such that any solution for which

$$
\operatorname{Re} \rho(0) \geq K(\gamma), \quad|\operatorname{Im} \rho(0)| \leq \frac{1}{18}, \quad|\operatorname{Im} \phi(0)| \leq \frac{\pi}{18}
$$

is defined for $0 \leq t \leq 2 \pi$ and satisfies there

$$
|\rho(t)-\rho(0)|<\gamma,|\phi(t)-\phi(0)|<\pi \gamma \text {. }
$$

Without loss of generality we may assume $\gamma<1 / 18$. Choose an integer $K(\gamma)$ so that

$$
\frac{P Q}{\omega^{4}(K(\gamma)-1 / 18)}<\frac{\gamma}{\pi} \text { and } K(\gamma)>\frac{19}{18} \text {, }
$$

where

$$
P=\max _{0 \leq t \leq 2 \pi}|p(t)|
$$

and

$$
Q=\max _{|\operatorname{Imu}| \leq \pi / 3}[|s(u)|+|c(u)|] .
$$

Any solution whose initial values satisfy the conditions is defined in some interval $[0, \beta)$. Write $\beta_{0}$ for the least positive $t$ for which one of

$$
\begin{aligned}
& \text { (i) }|\rho(t)-\rho(0)|=\gamma, \\
& \text { (ii) }|\phi(t)-\phi(0)|=\pi \gamma, \\
& \text { (iii) } t=2 \pi
\end{aligned}
$$

occurs. Then, for $0 \leq t \leq \beta_{0}$, we have

$$
|\operatorname{Im}(\rho(t) t+\phi(t))| \leq \frac{2}{18} \cdot 2 \pi+\frac{2 \pi}{18}=\frac{\pi}{3},
$$

and hence

$$
|\dot{\rho}(t)| \leq \frac{3}{2} \omega^{-4} P Q|\rho(t)|^{-1} \leq \frac{P Q}{2 \omega^{4}(K-1 / 18)}<\frac{\gamma}{2 \pi},
$$

which shows that 


$$
|\rho(t)-\rho(0)| \leq \int_{0}^{\beta_{0}}|\rho(u)| d u<\gamma
$$

and therefore that (i) cannot occur. Similarly (ii) cannot occur for $0 \leq t \leq \beta_{0}$ and $\beta_{0}$ must be determined by condition (iii), that is, $\beta_{0}=2 \pi$ and the asserted inequalities hold.

We shall use Lemma 8 in discussing derivative norms of $F_{\mu}$ and $G_{\mu}$. If $h(r, \theta)$ is $C^{s}$ in $A^{*}$ we define

$$
|h|_{s}=\sup \left|\left(\frac{\partial}{\partial r}\right)^{\sigma}\left(\frac{\partial}{\partial \theta}\right)^{\tau} h(r, \theta)\right|,
$$

where the supremum is taken over $A^{*}$ and all $\sigma+\tau \leq s$.

LEMMA 9. If $\delta, c_{0}$, and $l$ are given, there is an integer $\mu_{2}=\mu_{2}\left(\delta, c_{0}, l\right)$, such that for $\mu \geq \mu_{2}$,

$$
\begin{aligned}
& \left|E_{\mu}\right|_{0}+\left|G_{\mu}\right|_{0}<\delta, \\
& \left|E_{\mu}\right|_{2}+\left|G_{\mu}\right|_{2}<c_{0} .
\end{aligned}
$$

Choose $\gamma$ so that $5 \pi \gamma<\delta$ and

$$
\sup \left\{5 \pi \gamma \cdot \sigma ! \tau ! 18^{\sigma} \cdot\left(18 \pi^{-1}\right)^{\tau}\right\}<c_{0}
$$

and write $\mu_{2}=K(\gamma)$. Lemmas 5 to 8 show that if.

$$
\mu>K(\gamma),|\operatorname{Im} r| \leq 1 / 18,|\operatorname{Im} \theta| \leq \pi / 18,
$$

then $F_{\mu}(r, \theta)$ and $G_{\mu}(r, \theta)$ are defined, and hence analytic, and satisfy

$$
\left|F_{\mu}(r, \theta)\right|<3 \pi \gamma,\left|G_{\mu}(r, \theta)\right|<2 \pi \gamma,
$$

which give (5.3). If $r_{0}$ and $\theta_{0}$ are real, we see that $F_{\mu}(r, \theta)$ and $G_{\mu}(r, \theta)$ are analytic at least in

$$
\left|r-r_{0}\right| \leq 1 / 18,\left|\theta-\theta_{0}\right| \leq \pi / 18
$$

and hence, by using Cauchy's inequality for the coefficients in a (double) power series, that 


$$
\left|\left(\frac{\partial^{\sigma+\tau} F_{\mu}}{\partial r^{\sigma} \partial \theta^{\tau}}\right)\left(r_{0}, \theta_{0}\right)\right|<3 \pi \gamma \cdot \sigma ! \tau ! 18^{\sigma}\left(\frac{18}{\pi}\right)^{\tau}
$$

with a similar result for $G_{\mu}$. From these, (5.4) follows.

\section{Proofs of the theorems}

For the proof of our main result on boundedness we can work with a considerably simplified statement of Moser's theorem.

THEOREM A (Moser). Suppose that $F(r, \theta)$ and $G(r, \theta)$ have period $2 \pi$ in $\theta$ and that

$$
\left\{\begin{array}{l}
\theta_{1}=\theta+2 \pi r+F(r, \theta), \\
r_{1}=r+G(r, \theta)
\end{array}\right.
$$

sives a mapping of the annulus $1 \leq r \leq 2$ under which every closed curve encircling the pole intersects its image curve. Then there are an integer $\tau(1)$ and a real number $\delta_{0}$ such that from

$$
|F|_{0}+|G|_{0}<\delta_{0}
$$

and

$$
|F|_{2(1)}+|G|_{2(1)}<2 \pi
$$

it follows that the mapping has a closed invariant curve.

If this enunciation is compared with that of Theorem 1 in [9] it will be seen that we have left $\varepsilon$ unmentioned (that is, we do not try to specify the rotation number of our curve), that we have taken $a=1$, $b=2, \alpha(r)=2 \pi r$, and $c_{0}=2 \pi$, and that we have taken $s=1$ (that is, we do not seek a curve smoother than $C^{l}$ ).

Proof of Lemma 4. Since $T$ is area-preserving, any simple closed curve and its image under $T$ either intersect or have their interiors disjoint. For a curve in $A_{\mu}$ which encircles the origin the latter possibility is excluded for large $\mu$, say for $\mu \geq \mu_{3}$, and the two curves must intersect. It follows that for $\mu \geq \mu_{3}$ any simple closed curve in 
$A^{*}$ which encircles the pole meets its image under $T_{\mu}^{*}$.

If we write

$$
\mu_{1}=\max \left[\mu_{3}, \mu_{2}\left(\delta_{0}, 2 \pi, 2(1)\right)\right]
$$

then, for $\mu \geq \mu_{1}, T_{\mu}^{*}$ satisfies all the hypotheses of Theorem A.

Proof of Theorem 1. For each $\mu \geq \mu_{1}$ consider the invariant curve in $A_{\mu}$ and the tube $T_{\mu}$ arising from it. Since no trajectory can cross a tube we see that every solution of (1.3) is bounded.

Any point $(t, x, y)$ on the tube $T_{\mu}$ satisfies

$$
(\mu+1)^{4} \omega^{4}<y^{2}+x^{4}<(\mu+2)^{4} \omega^{4} .
$$

It follows that two points $\left(t_{i}, x_{i}, y_{i}\right), i=1,2$, which lie between $T_{\mu}$ and $T_{\mu+1}$ have

$$
(\mu+1) \omega<\left(y_{i}^{2}+x_{i}^{4}\right)^{\frac{3}{4}}<(\mu+3) \omega .
$$

In particular, if these points belong to the same trajectory we have, on this trajectory,

$$
\sup \left\{\dot{x}^{2}(t)+x^{4}(t)\right\}^{\frac{1}{4}}-\inf \left\{\dot{x}^{2}(t)+x^{4}(t)\right\}^{\frac{2}{4}} \leq 2 \omega .
$$

For a trajectory inside $T_{\mu_{1}}$ we have

$$
\sup \left\{\dot{x}^{2}(t)+x^{4}(t)\right\}^{\frac{3}{4}}-\inf \left\{\dot{x}^{2}(t)+x^{4}(t)\right\}^{\frac{3}{4}} \leq\left(\mu_{1}+2\right) \omega
$$

Hence the assertion about the oscillation of solutions is established, with $B=\left(\mu_{1}+2\right) \omega$, if we observe that $\mu_{1}$ depends on $p$ only through the definition of $K(\gamma)$ in Lemma 8, a definition which refers only to $P,=\max |p(t)|$.

For the discussion of Theorem 2 we do need to specify rotation numbers of curves.

THEOREM B (Moser). If $\beta>0$ then, for every irrational $\lambda$ in $(\beta, 1-\beta)$ which satisfies the diophantine condition that 


$$
|n \lambda-m|>\beta n^{-3 / 2}
$$

for all integers $m, n$ with $n>0$, there is a $\delta_{1}=\delta_{1}(\beta)$ such that if (6.1) satisfies

$$
\begin{array}{r}
|F|_{0}+|G|_{0}<\delta_{1}, \\
|F|_{Z(1)}+|G|_{Z(I)}<2 \pi,
\end{array}
$$

then there is a closed curve, I say, with the following properties:

(i) $\Gamma$ is invariant under the mapping,

(ii) the mapping induced on $\Gamma$ has rotation number $\lambda$,

(iii) $\Gamma$ can be parametrised

$$
\begin{aligned}
& \theta=\theta^{\prime}+q_{1}\left(\theta^{\prime}\right), \\
& r=\lambda+q_{2}\left(\theta^{\prime}\right),
\end{aligned}
$$

with $q_{1}$ and $q_{2}$ both $c^{1}$ functions of period $2 \pi$,

(iv) $\left|q_{1}\right|_{1}+\left|q_{2}\right|_{1}<\beta$, and

(v) the mapping induced on $\Gamma$ is given by

$$
\theta_{1}^{\prime}=\theta^{\prime}+2 \pi \lambda \text {. }
$$

If this enunciation is compared with Theorem 1 and the remark following it in [9] it will be seen that $\varepsilon$ is now mentioned and renamed $2 \pi \beta$, and that, to avoid clashes of notation with the work here, $\omega, p, q$, and $\delta_{0}$ have been changed to $2 \pi(1+\lambda), q_{1}, q_{2}$, and $\delta_{1}$ respectively.

Proof of Theorem 2. If $\beta$ is small the set, $\Lambda(\beta)$ say, of $\lambda$ in $(\beta, 1-\beta)$ satisfying the diophantine condition (6.2) has positive measure, since it is greater than

$$
1-2 \beta-2 \beta \sum_{1}^{\infty} n^{-3 / 2} \text {. }
$$

Write

$$
\Lambda=\underset{\beta>0}{U} \Lambda(\beta)
$$


Evidently $\mathrm{m} \Lambda=1$.

If $\lambda \in \Lambda$, there is a $\beta$ such that $\lambda \in \Lambda(\beta)$. If we imitate the proof of Lemma 4 and write

$$
\mu^{*}=\max \left[\mu_{3}, \mu_{2}\left(\delta_{1}(B), 2 \pi, Z(1)\right)\right],
$$

we see that, for $\mu \geq \mu^{*}$, there is a curve $\Gamma_{\mu}$ in $A_{\mu}$ which is invariant under $T$ and has rotation number $\lambda$.

The property $(v)$ shows that the mapping induced by $T$ is ergodic. It follows, if we consider the differential equation induced on $T_{\mu}$ by (1.3) and use Bohl's Theorem 5.3 from [3] page 414, that any trajectory arising from a point of $\Gamma_{\mu}$ is almost-periodic, with basic frequencies $2 \pi$ and $2 \lambda \pi$

For the proof of Theorem 3 it will be convenient to have Poincaré's Last Geometric Theorem presented for a strip, as in Birkhoff's proof.

THEOREM C (Birkhoff). Suppose that $W$ is a topological mapping of the strip

$$
-\infty<x<\infty, \quad a^{2} \leq y \leq b^{2}
$$

onto itself which has the following properties:

(i) $W$ has a positive integral invariant,

(ii) $W$ has period $2 \pi$ in $x$,

(iii) $W$ regresses points on $y=a^{2}$ and advances points on $y=b^{2}$.

Then, either

(a) $W$ has an infinity of inequivalent fixed points, or

(B) $W$ has a finite number of inequivalent fixed points, including two at which the rotations are of opposite signs.

(If we write $R$ for the mapping of the strip defined by

$$
R(x, y)=(x+2 \pi, y),
$$

we say $z_{1},=\left(x_{1}, y_{1}\right)$, and $z_{2}$ are equivalent if, for some integer $v$, 
$R^{\nu} z_{1}=z_{2}$. ) Theorem $\mathrm{C}$ is proved in [1] (or, in the weak form that $W$ has "in general" at least two fixed points, in [2], pages 165-169).

Proof of Theorem 3. Consider two curves $\Gamma_{K}$ and $\Gamma_{K+1}$, invariant under $T$, which lie in consecutive annuli $A_{K}$ and $A_{K+1}$ and have the same irrational rotation number modulo 1 . At least for large $k$, say for $k \geq K^{*}$, we can say, by analogy with the comparison equation (3.1), that their rotation numbers differ by $I$, being $\lambda$ and $\lambda+1$ say. We shall show that in the ring between $\Gamma_{K}$ and $\Gamma_{K+1}$ there lie at least $2 m \phi(m)$ fixed points of (proper) order $m$ of $T$.

By any convenient diffeomorphism take this ring into the circular ring $0<a \leq r \leq b$,

and by writing

$$
x=\theta, y=r^{2}
$$

take the circular ring into the infinite strip $a^{2} \leq y \leq b^{2}$. On the circular ring and the infinite strip we see that $T$ is represented by a homeomorphism $U$ and a periodic homeomorphism $V$ respectively. Corresponding to the area-preserving property of $T$, each has a positive integral invariant.

If $z$ is any point of the strip, write $F z$ for the corresponding point of the non-circular ring between $A_{K}$ and $A_{K+1}$. Evidently, for any integers $m$ and $s$,

$$
T^{m} F=F V^{m} \text { and } F R^{s}=F \text {, }
$$

and if $z$ is fixed under $R^{S} V^{m}$ then $F_{z}$ is fixed under $T^{m}$. Further, $F_{z}=F_{z^{\prime}}$, if and only if $z$ and $z^{\prime}$ are equivalent.

Consider any positive integer $m$. Under $T^{m}$ and $U^{m}$ the rings have their inner and outer boundaries rotated, on an average, through $m \lambda$ and $m(1+\lambda)$ revolutions, and, correspondingly, $v^{m}$ advances points on $y=a^{2}$ through an average distance $m \lambda .2 \pi$ and those on $y=b^{2}$ through $m(1+\lambda) \cdot 2 \pi$. We see that, for any integer $k$ with $0<k \leq m$, the 
compound mapping

$$
R^{-k-[m \lambda]} V^{m}
$$

gives average advances $(-k+\{m \lambda\}) .2 \pi$ and $(m-k+\{m \lambda\}) \cdot 2 \pi$, that is, it regresses points on $y=a^{2}$ and advances those on $y=b^{2}$. (Here $\{m \lambda\},=m \lambda-[m \lambda]$, is the fractional part of $m \lambda$, and not 0 since $\lambda$ is irrational.) It follows that $(\alpha)$ or $(\beta)$ of Theorem $C$ holds for this mapping.

Whether $(\alpha)$ or $(\beta)$ occurs, suppose that $z_{1}$ is a fixed point, that is,

$$
V_{z_{1}}=R^{k+[m \lambda]} z_{1}
$$

We shall show that if $k+[m \lambda]$ is prime to $m$ then the points

$$
F_{z_{1}}, T F_{z_{1}}, \ldots, T^{m-1} \mathrm{Fz}_{1}
$$

are distinct. Suppose if possible that $T^{2} F_{z_{I}}=F_{z_{I}}$, that is, that

$$
F V^{2} z_{1}=F z_{1}
$$

with $0<\tau<m$. Since we know $F_{z_{1}}$ is fixed under $T^{m}$ we must have $m=B Z$, where $B$ is an integer. From (6.5) we have that there is an integer $\nu$ for which

$$
v_{z_{1}}^{z_{1}}=R^{v} z_{1}
$$

from which we could deduce

$$
v_{z_{1}}^{m}=R_{z_{1}}^{\beta v_{2}}
$$

which is inconsistent with (6.3). Hence the points (6.4) are distinct and none can be fixed under $T^{l}$ with $0<Z<m$. We know each is fixed under $T^{m}$, and thus have $m$ fixed points of $T$ of order $m$.

If it is $(\alpha)$ which holds, we have an infinity of fixed points of order $m$. If it is $(\beta)$, we can suppose without loss of generality that $z_{1}$ has 
positive rotation and assert that there is a $z_{2}$ having negative rotation. We evidently obtain at least $2 m$ fixed points of order $m$ corresponding to this value of $k$ and at least $2 m \phi(m)$ altogether.

If we restate this for periodic solutions of (1.3) and note that $\Gamma_{K}$ and $\Gamma_{K+1}$ lie in the annulus specified, we have the enunciated statement. $\square$

\section{The relation of this work to Littlewood's}

In Littlewood's example of an equation of the type (1.1) which has an unbounded solution, both $g$ and $p$ are discontinuous. This is obviously irrelevant for $p$ but might seem to matter for $g$; for definiteness I show here that his construction of $g$ can be modified so that the function is in $C^{\infty}$. The existence of such a $g$ is quite consistent with the suggestion that (1.1) with $g$ many times differentiable, on whose derivatives we could place strong bounds, would be accessible to the methods of this paper since we can claim we have indirect evidence that any modification of Lit+lewood's $g$ which preserved an unbounded solution of the equation would, even if $c^{\infty}$ or $c^{\omega}$ on the real axis, have some very large derivatives.

To modify Littlewood's $g$ to a $c^{\infty}$ function, we notice that, in the notation of p. 503 of [5], $\Gamma_{2 n}(y)$ is continuous in $R_{2 n-1}=\left[y_{2 n-1}, y_{2 n+1}\right]$ and defined in each half of this interval as a polynomial. We can modify this definition in neighbourhoods of $y_{2 n-1}$, $\frac{1}{2}\left(y_{2 n-1}+y_{2 n+1}\right)$, and $y_{2 n+1}$ so as to make $\Gamma_{2 n}$ infinitely differentiable with

$$
\Gamma_{2 n}^{(r)}\left(y_{2 n-1}\right)=\Gamma_{2 n}^{(r)}\left(y_{2 n+1}\right)=0
$$

for all $r$, while keeping the modification small enough to allow $c_{2 n}$ to be chosen. Then, defining $g$ as before in $R_{2 n-1}$ by

$$
g(y)=d\left[y^{4}+c_{2 n} \Gamma_{2 n}(y)\right] / d y
$$

we have that it is $C^{\infty}$ in that interval and, because of (7.1), that, 
however $c_{2 n}$ and $c_{2 n+2}$ are chosen, the derivatives are continuous as we cross into $R_{2 n+1}$.

\section{References}

[1] George D. Birkhoff, "An extension of Poincaré's last geometric theorem", Acta Math. 47 (1926), 297-311; see also George David Birkhoff, Collected mathematical papers, Vol. II, 252-266 (Amer. Math. Soc., New York, 1960).

[2] George D. Birkhoff, Dynamical systems (Colloquium Publications, 9. Amer. Math. Soc., New York, 1927; reprinted Amer. Math. Soc., Providence, Rhode Island, 1966).

[3] Earl A. Coddington and Norman Levinson, Theory of ordinary differential equations (McGraw-Hill, New York, Toronto, London, 1955).

[4] J.E. Littlewood, "Unbounded solutions of $\ddot{y}+g(y)=p(t) ", J$. London Math. Soc. 41 (1966), 491-496.

[5] J.E. Lit+lewood, "Unbounded solutions of an equation $\ddot{y}+g(y)=p(t)$, with $p(t)$ periodic and bounded, and $g(y) / y \rightarrow \infty$ as $y \rightarrow \pm \infty$ ", J. London Math. Soc. 41 (1966), 497-507.

[6] John E. Littlewood, Some problems in real and complex analysis (Heath, Lexington, Massachusetts, 1968).

[7] G.R. Morris, "A differential equation for undamped forced non-linear oscillations. I", Proc. Cambridge Philos. Soc. 51 (1955), 297-312.

[8] G.R. Morris, "A differential equation for undamped forced non-linear oscillations. II", Proc. Cambridge Philos. Soc. 54 (1958), 426-438.

[9] J. Moser, "On invariant curves of area-preserving mappings of an annulus", Nachr. Akad. Wiss. Göttingen Math.-Phys. K2. II 1962, $1-20$. 
[10] E.T. Whittaker and G.N. Watson, A course of modern analysis: an introduction to the general theory of infinite processes and of analytic functions; with an account of the principal transcendental functions, fourth edition (Cambridge University Press, Cambridge, 1935).

Department of Mathematics,

University of New England,

Armidale,

New South Wales. 